\title{
A Rat Model of Intracerebral Hemorrhage Permitting Hematoma Aspiration plus Intralesional Injection
}

\author{
Yan-Hua SANG ${ }^{1,2)}$, Yu-Xiang LIANG ${ }^{2,3)}$, Ling-Guang LIU' ${ }^{1)}$, Rutledge G. ELLIS-BEHNKE 2,3,6,7), \\ Wu-Tian WU ${ }^{2,3,4)}$, Kwok-Fai SO ${ }^{2,3,5)}$, and Raymond Tak-Fai CHEUNG ${ }^{1,5)}$ \\ ${ }^{1)}$ Department of Medicine, Li Ka Shing Faculty of Medicine, The University of Hong Kong, Hong Kong, China \\ ${ }^{2)}$ Department of Anatomy, Li Ka Shing Faculty of Medicine, The University of Hong Kong, Hong Kong, China \\ ${ }^{3}$ State Key Laboratory of Brain and Cognitive Sciences, Li Ka Shing Faculty of Medicine, The University of Hong \\ Kong, Hong Kong, China \\ ${ }^{4)}$ Research Centre of Reproduction, Development and Growth, Li Ka Shing Faculty of Medicine, The University of \\ Hong Kong, Hong Kong, China \\ ${ }^{5)}$ Research Centre of Heart, Brain, Hormone and Healthy Aging, Li Ka Shing Faculty of Medicine, The University \\ of Hong Kong, Hong Kong, China \\ ${ }^{6)}$ Department of Brain and Cognitive Sciences, Massachusetts Institute of Technology, Cambridge, MA, USA \\ 7) Department of Ophthalmology, Medical Faculty Mannheim, Ruprecht-Karls-University, Heidelberg, Germany
}

\begin{abstract}
A combination of hematoma aspiration and local delivery of chemicals may be more effective than either therapy in intracerebral hemorrhage $(\mathrm{ICH})$. The aim of the present study was to develop a rat model of hematoma aspiration plus intralesional injection after $\mathrm{ICH}$. ICH was induced in adult Sprague-Dawley rats by an intrastriatal injection of bacterial collagenase IV. Hematoma aspiration was performed $3.5 \mathrm{~h}$ after ICH onset. Following aspiration, normal saline was injected into the lesion cavity. Hematoma aspiration with or without subsequent saline injection significantly reduced the hematoma volume, lesion volume, and perihematomal neutrophil infiltration. Hematoma aspiration plus subsequent intralesional injection is simple, feasible, and safe. This $\mathrm{ICH}$ model can be used to assess the effectiveness of hematoma removal plus local delivery of chemicals.

Key words: animal model, hematoma aspiration, intracerebral hemorrhage, intralesional injection, rat
\end{abstract}

\section{Introduction}

Intracerebral hemorrhage (ICH) is usually caused by rupture of a cerebral vessel or aneurysm [18]. ICH is the least treatable type of stroke and a major public health problem, affecting two million people worldwide each year [23]. Management of ICH is largely supportive, and the outcome remains poor [3, 7, 28]. Several therapeutic approaches for ICH are being evaluated, including stereotactic minimally invasive aspiration, neuroprotective drugs, and neural stem cell transplantation. Clinical trials addressing a single pathogenic factor, such as hemorrhage volume or hematoma enlargement, have been successfully conducted, but clinical benefit cannot be shown $[12,13,15,18]$. The negative results suggest that a combination of hematoma removal and local delivery of chemicals may be more effective [26].

Hematoma removal may alleviate the mass effect, lessen the damage caused by release of lysed blood products, and prevent the secondary pathological processes. The European Stroke Initiative Guidelines recommend stereotactic minimally invasive methods to remove a deep hematoma within $12 \mathrm{~h}$ of ICH onset [22]. A trial of stereotactic treatment of intracerebral hematoma by means of a plasminogen activator reported a significant reduction in the hematoma volume by $10-20 \%$ via ste- 
reotactic aspiration [24]. Nevertheless, there was no difference in survival at 180 days. Our current knowledge concerning the potential benefit of a combination of hematoma aspiration and local delivery of chemicals is limited partly because of a lack of suitable animal models. The purpose of this study was to establish a rat model incorporating stereotactic minimally invasive aspiration plus intralesional injection using an established ICH model [19].

\section{Materials and Methods}

\section{Subjects and experimental groups}

Experimental protocols were approved by the Committee on the Use of Live Animals in Teaching and Research, the University of Hong Kong. A total of 67 male Sprague-Dawley (SD) rats, 11-13 weeks old and weighing between 400 and $500 \mathrm{~g}$, were used in this study. They were provided by the Laboratory Animal Unit, the University of Hong Kong, and were reared in a temperaturecontrolled room with standard chow and water supply. The rats were divided into 3 groups: the ICH only group $(\mathrm{n}=20)$, with induction of ICH and sham hematoma aspiration; the aspiration only group $(\mathrm{n}=26)$, with induction of ICH and subsequent hematoma aspiration; and the saline group $(\mathrm{n}=21)$, with induction of $\mathrm{ICH}$, hematoma aspiration, and intralesional injection of saline. Hematoma volume, lesion volume, and brain swelling were assessed 1 day after ICH induction. Histological analysis was performed 3 days after ICH induction. Prior to sacrifice, functional deficits were assessed by a blinded observer.

\section{Intracerebral hemorrhage}

Experimental ICH was induced via an intrastriatal injection of type IV collagenase $[11,17]$. In brief, the rat was anesthetized using an intraperitoneal (i.p.) injection of sodium pentobarbital $(55 \mathrm{mg} / \mathrm{kg})$ and placed in a stereotaxic frame. A burr hole with a diameter of $2 \mathrm{~mm}$ was drilled along the left coronal suture at $3.0 \mathrm{~mm}$ lateral to the bregma. A 30-gauge $(\mathrm{G})$ needle was inserted into the left striatum with its tip at $0.2 \mathrm{~mm}$ anterior to the bregma, $3 \mathrm{~mm}$ lateral to the midline, and $6 \mathrm{~mm}$ underneath the dural surface. ICH was induced by a slow injection of $0.2 \mathrm{U}$ collagenase IV (Sigma-Aldrich, St. Louis, MO, USA) in $1.0 \mu \mathrm{l}$ saline into the left striatum over $10 \mathrm{~min}$. After injection, the needle was left in place for $5 \mathrm{~min}$. The burr hole was sealed with bone wax, and the incision was sutured. Rectal temperature was maintained around $37 \pm 0.5^{\circ} \mathrm{C}$ using a rectal thermostat probe and a thermostatically regulated heating pad (DC Temperature Control System, FHC Inc., Bowdoin, ME, USA). The right femoral artery was cannulated with a PE-50 catheter (Portex Ltd., Hythe, UK) for monitoring of arterial blood pressure and heart rate using a PowerLab 16/35 Data Acquisition Systems (AD Instruments, Milford, MA, USA) as well as for measurement of blood glucose levels.

\section{Hematoma aspiration plus intralesional injection}

In order to determine the best time for hematoma aspiration, three different time points were studied: $2,3.5$, and $6 \mathrm{~h}$ after collagenase injection. The rats were randomly allocated to one of the above-mentioned time points ( $\mathrm{n}=4$ per time point). In brief, the rats were reanesthetized with pentobarbital $(55 \mathrm{mg} / \mathrm{kg}$, i.p.) and placed in a stereotactic frame. Aspiration was accomplished by gentle suction with a syringe attached to a 23-G needle placed at the center of the hematoma. Four attempts were made over $15 \mathrm{~min}$. Care was taken to avoid blockade of the needle by the hematoma or bone wax. The volume of aspirated blood was measured using a $100 \mu \mathrm{l}$ pipette. The aspirated blood was smeared onto SuperFrost Plus slides (Menzel-Glaser, Braunschweig, Germany). After fixation, the slides were stained with hematoxylin and eosin (HE) and then examined under a microscope.

Hematoma aspiration at $3.5 \mathrm{~h}$ after ICH was used in subsequent experiments. The saline injection group received $20 \mu \mathrm{l}$ saline via a $25-\mathrm{G}$ needle at an infusion rate of $500 \mu \mathrm{l}$ per hour immediately following hematoma aspiration. In the ICH only group, a $23-\mathrm{G}$ needle was placed at the center of the hematoma for 4 attempts over 15 min without any suction attempts. After injection or sham injection, the burr hole was sealed with bone wax, and the incision was sutured.

\section{Hematoma volume}

Hematoma volume was quantified at $24 \mathrm{~h}$ using a spectrophotometric assay [5, 10, 17]. In brief, hemispheric brain tissue minus the olfactory bulbs and cerebellum was acquired following transcardial perfusion. The tissue was homogenized in $3 \mathrm{ml} 0.01 \mathrm{M} \mathrm{PBS}$, and this was followed by $1 \mathrm{~min}$ of sonication on ice. After centrifugation at $12,000 \mathrm{~g}$ for $30 \mathrm{~min}, 100 \mu 1$ supernatant was reacted with $400 \mu 1$ Drabkin's reagent (Sigma-Al- 
drich) for $15 \mathrm{~min}$. The absorbance reading minus the background reading at $540 \mathrm{~nm}$ was determined with a spectrophotometer (BioTek Instruments Inc., Winooski, VT, USA). Using a previously determined curve from known hemoglobin contents, the hematoma volume in the perfused brain was quantified.

\section{Lesion volume}

Rats were sacrificed 1 day after ICH induction to determine lesion volume. In brief, the rats were deeply anesthetized with sodium pentobarbital $(80 \mathrm{mg} / \mathrm{kg}$, i.p.) and perfused transcardially with saline and then $4 \%$ paraformaldehyde in $0.1 \mathrm{M}$ phosphate buffer (PB) for $20 \mathrm{~min}$. The brain was sliced into $1 \mathrm{~mm}$-thick coronal slices. Images of the brain slices were taken using a digital camera and analyzed with the ImageJ software (National Institutes of Health, Bethesda, MA, USA) by a blinded observer. Lesion volume was calculated by the lesion area of each slice multiplied by the slice thickness [2].

\section{Tissue preparation and immunohistochemical analysis}

Rats were deeply anesthetized with sodium pentobarbital $(80 \mathrm{mg} / \mathrm{kg}$, i.p.) 3 days after ICH induction and perfused transcardially with saline and then $4 \%$ paraformaldehyde in $0.1 \mathrm{M}$ PB for $20 \mathrm{~min}$. The brain was postfixed in $4 \%$ paraformaldehyde overnight at $4{ }^{\circ} \mathrm{C}$ before being placed in $0.1 \mathrm{M} \mathrm{PB}$ containing $30 \%$ sucrose. Brain sections at $30 \mu \mathrm{m}$ were obtained between $3 \mathrm{~mm}$ anterior to the bregma and $4 \mathrm{~mm}$ posterior to the bregma using a cryostat at $-18^{\circ} \mathrm{C}$. Brain sections were affixed on $\mathrm{Su}-$ perFrost Plus slides, air-dried overnight, and stored for immunohistochemical (IHC) studies.

After blocking with $10 \%$ normal goat serum in phosphate buffered saline (PBS), the brain sections were incubated with the primary antibodies at $4^{\circ} \mathrm{C}$ overnight. Neutrophils were detected with rabbit anti-myeloperoxidase (MPO, 1:200, Dako, Glostrup, Denmark). The brain sections were then rinsed and incubated with biotinylated goat anti-rabbit antibody $(1: 200$, Vector, Burlingame, CA, USA) for $2 \mathrm{~h}$. Avidin-biotin complex solution was used to amplify the IHC signal, which was visualized using diaminobenzidine as the chromogen. Finally, the brain sections were coverslipped for examination under a light microscope.

\section{Behavioral test}

Rats were subjected to a modified limb placing test
(MLPT) 1 day, 3 days, 7 days, and 2 weeks after ICH by a blinded observer [21]. Firstly, the rats were suspended at $10 \mathrm{~cm}$ above a table, and the stretch of the forelimbs toward the table was scored as follows: 0 point for normal stretch and 1 point for abnormal flexion. Next, the rats were positioned along the table edge, and their forelimbs were suspended over the edge and allowed to move freely. Each limb (forelimb, second task; hind limb, third task) was pulled down gently, and the retrieval and placement were checked. Finally, the rats were placed toward the table edge to check for lateral placement of the forelimbs. The last three tasks were scored in the following manner: 0 point for normal performance, 1 point for performance with a delay $(2 \mathrm{~s})$ or incomplete performance, and 2 points for no performance. A score of seven points indicates maximal neurological deficit, and a score of 0 point indicates normal performance.

\section{Statistical analysis}

All numerical values are expressed as the mean \pm standard deviation. Data were analyzed with a two sample $t$-test or one-way analysis of variance followed by Turkey's test, as appropriate. A $P$ value of 0.05 or less was taken to infer statistical significance.

\section{Results}

\section{Physiological parameters}

There were no significant differences among the ICH only group, aspiration only group, and saline group with regard to body weight (data not shown), blood pressure, heart rate, rectal temperature, and blood glucose levels upon induction of ICH (Table 1).

\section{ICH plus hematoma aspiration}

Intrastriatal injection of $0.2 \mathrm{U}$ collagenase IV caused a medium-sized hematoma. It was generally restricted to the dorsolateral striatum (caudate putamen and globus pallidus; Fig. 1). The hematoma could be aspirated $2 \mathrm{~h}$ $(\mathrm{n}=4)$ and $3.5 \mathrm{~h}(\mathrm{n}=4)$ but not $6 \mathrm{~h}(\mathrm{n}=4)$ after onset of ICH. The volume of aspirated blood ranged from 20 to $40 \mu \mathrm{l}$. HE staining of the aspirated blood showed red blood cells, neutrophils, and islands of fragmented striatum (Fig. 2).

\section{Hematoma volume and lesion volume}

Following hematoma aspiration at $3.5 \mathrm{~h}$ after onset, saline was injected into the lesion in the saline group. 
Table 1. Physiological parameters upon induction of ICH

\begin{tabular}{lcccc}
\hline \multicolumn{1}{c}{ Groups } & MAP $(\mathrm{mmHg})$ & HR $(\mathrm{bpm})$ & Temperature $\left({ }^{\circ} \mathrm{C}\right)$ & Glucose $(\mathrm{mmol} / \mathrm{l})$ \\
\hline ICH only & $85.8 \pm 12.3$ & $324.5 \pm 47.9$ & $37.5 \pm 0.2$ & $6.9 \pm 1.7$ \\
Aspiration only & $85.7 \pm 11.5$ & $331.6 \pm 48.3$ & $37.4 \pm 0.2$ & $7.3 \pm 1.1$ \\
Saline & $84.2 \pm 12.3$ & $332.3 \pm 54.2$ & $37.5 \pm 0.2$ & $7.5 \pm 1.3$ \\
\hline
\end{tabular}

$\mathrm{n}=6$ per group. HR, heart rate; MAP, mean arterial pressure. There were no significant differences among the three groups.

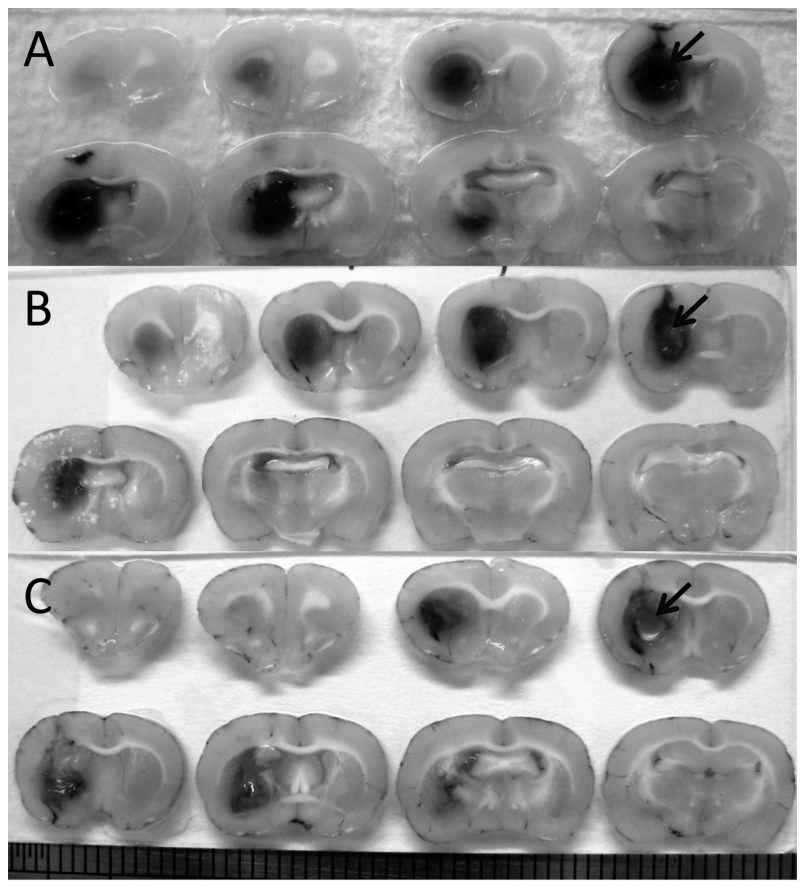

Fig. 1. Hemorrhagic brain lesion 1 day after collagenase IV injection. (A) ICH only. (B) ICH with aspiration. (C) ICH with aspiration and intralesional injection of saline. Arrows show the needle entry section.

One day after ICH induction, the brain of both the aspiration only group and saline group had a cavity within the center of the lesion (Fig. 1). The hematoma volume was $29.3 \pm 1.7 \mu 1$ in the ICH only group $(n=5), 20.7 \pm$ $3.1 \mu 1$ in the aspiration only group $(n=5)$ and $19.9 \pm 4.0$ $\mu \mathrm{l}$ in the saline group $(\mathrm{n}=5)$. The hematoma volume was significantly reduced by aspiration (Fig. 3). However, it was not significantly different between the aspiration only group and saline group. The lesion volume was 31.5 $\pm 3.5 \mu 1$ in the ICH only group $(\mathrm{n}=5), 24.4 \pm 5.7 \mu \mathrm{l}$ in the aspiration only group $(\mathrm{n}=6)$, and $21.6 \pm 4.2 \mu 1$ in the saline group $(\mathrm{n}=5)$. The lesion volume was significantly reduced by aspiration but not further reduced by saline injection (Fig. 3).

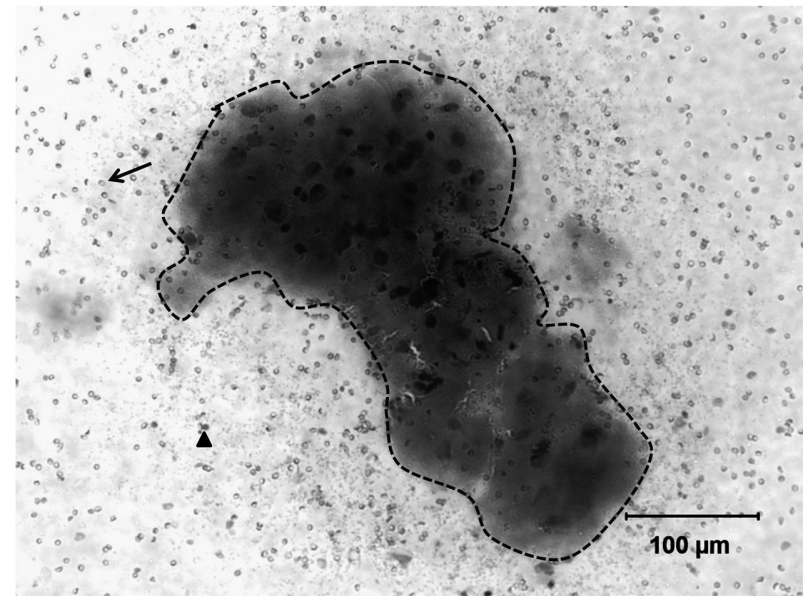

Fig. 2. HE staining of the aspirated blood $3.5 \mathrm{~h}$ after ICH induction showing red blood cells (arrow), neutrophils (arrowhead), and an island of fragmented striatum (dotted line). HE, hematoxylin and eosin.

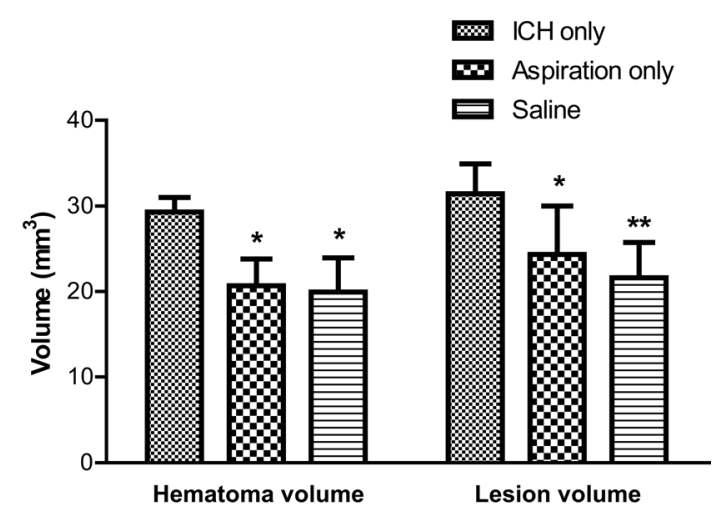

Fig. 3. Hematoma volume and lesion volume quantified 1 day after ICH induction. Aspiration with or without saline injection was performed at $3.5 \mathrm{~h}$ after onset. $* P<0.05$ and $* * P<0.01$ when compared with the ICH only group.

\section{Neutrophil infiltration}

MPO staining revealed a high density of immunopositive cells within and around the hemorrhagic lesion 3 days after ICH induction (Fig. 4). Aspiration of the hematoma reduced the number of MPO-positive cells in 
A

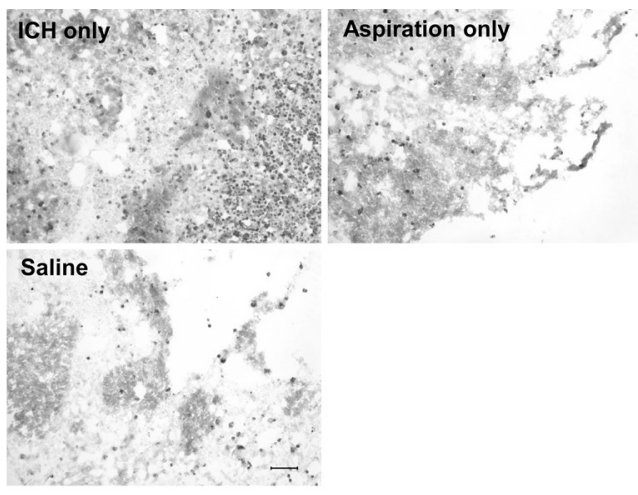

B

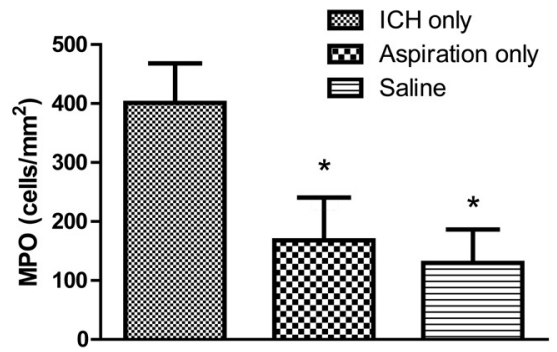

Fig. 4. Brain MPO staining 3 days after ICH induction. (A) Images show MPO staining in the perihematomal area. (B) Quantification analysis. ${ }^{*} P<0.05$ when compared with the ICH only group. Scale bar $=50 \mu \mathrm{m}$ in A.

the perihematomal area when compared with the ICH only group ( $409.0 \pm 60.2$ cells $\left./ \mathrm{mm}^{2}, \mathrm{n}=4\right)$, and the number of MPO-positive cells was similar in both the aspiration only group $\left(158.0 \pm 71.5 \mathrm{cells} / \mathrm{mm}^{2}, \mathrm{n}=4\right)$ and saline group (119.6 \pm 60.5 cells $/ \mathrm{mm}^{2}, \mathrm{n}=4$, Fig. 4$)$.

\section{Functional deficit}

All rats survived the surgery and completed the behavioral test. The rats developed significant limb placing deficits $1,3,7$, and 14 days after ICH induction. Hematoma aspiration did not affect the behavioral outcome at all time points (Fig. 5). The respective MLPT scores were $6.8 \pm 0.3,6.9 \pm 0.2,6.4 \pm 0.5$, and $5.6 \pm 0.5$ in the ICH only group $(n=5), 6.8 \pm 0.3,6.6 \pm 0.3,6.5 \pm 0.3$, and $6.1 \pm 0.1$ in the aspiration only group $(\mathrm{n}=5)$, and 6.9 $\pm 0.2,6.7 \pm 0.2,6.5 \pm 0.5$, and $6.0 \pm 0.7$ in the saline group $(\mathrm{n}=6)$.

\section{Discussion}

This study successfully established a rat ICH model incorporating hematoma aspiration plus intralesional injection. Following hematoma aspiration, intralesional

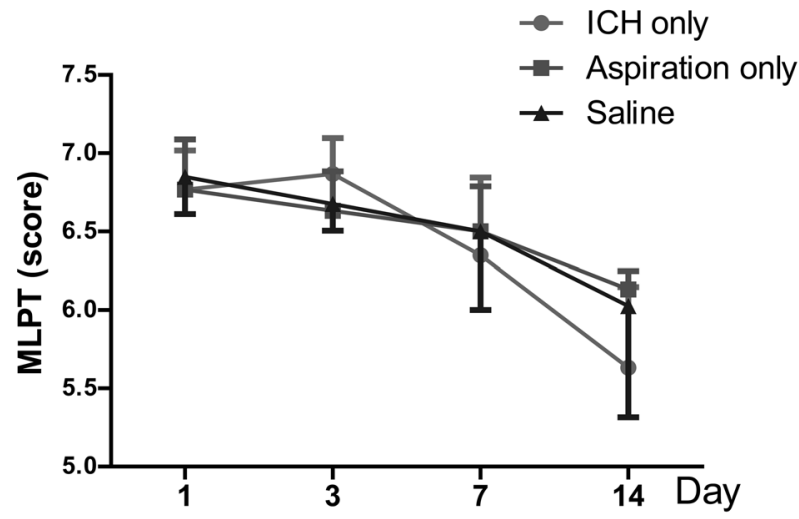

Fig. 5. Functional deficit score. Aspiration with or without saline injection was performed at $3.5 \mathrm{~h}$ after onset. There were no significant differences in functional recovery among all groups $1,3,7$, and 14 days after ICH induction.

injection is a simple and feasible procedure. The hematoma was aspirated $3.5 \mathrm{~h}$ after ICH onset without using a thrombolytic drug. Hematoma aspiration and/or saline injection reduced the hematoma volume, lesion volume, and perihematomal neutrophil infiltration in the rat.

In a previous study, ICH was induced by collagenase injection, and streptokinase was locally administered at $4 \mathrm{~h}$ after ICH onset to lyse the hematoma to permit hematoma aspiration at $5 \mathrm{~h}$ after ICH onset [1]. Slight improvement of global behavior was observed at 4 weeks after hematoma aspiration, but there was no significant difference in the skilled forelimb function at 7 weeks. The use of thrombolytic agents to lyse the hematoma is being investigated [14], and the adverse effects of thrombolytic agents on the brain parenchyma and vasculature are our concerns [9, 25, 27]. In the present study, hematoma aspiration was performed without using a thrombolytic agent, and intralesional injection of saline was performed. Without using any thrombolytic agent, the hematoma could be aspirated between 2 and $3.5 \mathrm{~h}$ after collagenase injection, and aspiration was not feasible 6 $\mathrm{h}$ after ICH onset. This finding is consistent with blood clotting within $4 \mathrm{~h}$ after collagenase injection in the rat [6]. In clinical practice, it is likely that hematoma aspiration is feasible for a few hours after ICH onset prior to clot formation.

To maintain minimal disturbance of the intracranial pressure (ICP), a volume of saline of around two thirds of the original hematoma volume, i.e., $20 \mu \mathrm{l}$, was injected into the lesion immediately following hematoma aspiration. Although the ICP was not measured directly, 
there were no significant differences in blood pressure and heart rate among the three groups.

In our study, we used normotensive rats, and hematoma aspiration significantly reduced the hematoma volume. However, this may not be the case clinically since hypertension is the most important risk factor for ICH [4]. Our previous study showed that elevated blood pressure increased the hematoma volume in rats [20]. Therefore, the effects of hematoma aspiration in hypertensive ICH rats need to be studied in the future.

Inflammation occurs after ICH and is characterized by infiltration of neutrophils and macrophages as well as activation of microglia [8]. Both the hematoma and damaged brain tissue liberate chemotactic factors, including thrombin, which attract neutrophils from the blood stream to migrate into the brain tissue around the hematoma [16]. In the present study, hematoma aspiration significantly lessened the toxic blood components. Thus, neutrophil infiltration in the perihematomal area was reduced following hematoma aspiration. Nevertheless, the reduction in hematoma volume, lesion volume, and neutrophil infiltration did not translate into functional improvement up to 14 days after ICH induction. It is possible that incorporation of another treatment strategy or a greater reduction in lesion volume is needed to achieve a functional benefit. In addition, a longer period of observation may be required. Finally, the surgical aspiration was performed over $15 \mathrm{~min}$ via a burr hole; the adverse effects of local injury by this burr-hole stereotaxic aspiration on the perifocal or diffuse brain edema and the functional score need to be studied in the future.

In summary, hematoma aspiration plus intralesional injection is a simple, minimally invasive technique that can be performed without craniotomy. This model may be useful for evaluation of combinational treatment strategies in ICH. Early removal of the hematoma not only lessens this detrimental factor but also gives room for local administration of a neuroprotective agent.

\section{Acknowledgments}

This work was supported by the General Research Fund of the Research Grants Council (HKU763109M) and the Committee on Research and Conference Grants (200707176018, 200807176052, 201007176091).

\section{References}

1. Altumbabic, M., Peeling, J., and Del Bigio, M.R. 1998. Intracerebral hemorrhage in the rat: effects of hematoma aspiration. Stroke 29: 1917-1922, discussion 1922-1923. [Medline] [CrossRef]

2. Auriat, A., Plahta, W.C., Mcgie, S.C., Yan, R., and Colbourne, F. 2005. 17 beta-estradiol pretreatment reduces bleeding and brain injury after intracerebral hemorrhagic stroke in male rats. J. Cereb. Blood Flow Metab. 25: 247-256. [Medline] [CrossRef]

3. Broderick, J., Connolly, S., Feldmann, E., Hanley, D., Kase, C., Krieger, D., Mayberg, M., Morgenstern, L., Ogilvy, C.S., Vespa, P., and Zuccarello, M. 2007. Guidelines for the management of spontaneous intracerebral hemorrhage in adults: 2007 update: a guideline from the American Heart Association/American Stroke Association Stroke Council, High Blood Pressure Research Council, and the Quality of Care and Outcomes in Research Interdisciplinary Working Group. Circulation 116: e391-e413. [Medline] [CrossRef]

4. Brott, T., Thalinger, K., and Hertzberg, V. 1986. Hypertension as a risk factor for spontaneous intracerebral hemorrhage. Stroke 17: 1078-1083. [Medline] [CrossRef]

5. Choudhri, T.F., Hoh, B.L., Solomon, R.A., Connolly, E.S. Jr., and Pinsky, D.J. 1997. Use of a spectrophotometric hemoglobin assay to objectively quantify intracerebral hemorrhage in mice. Stroke 28: 2296-2302. [Medline] [CrossRef]

6. Del Bigio, M.R., Yan, H.J., Buist, R., and Peeling, J. 1996. Experimental intracerebral hemorrhage in rats. Magnetic resonance imaging and histopathological correlates. Stroke 27: 2312-2319, discussion 2319-2320. [Medline] [CrossRef]

7. Gere, J., Minier, D., Osseby, G.V., Couvreur, G., Moreau, T., Ricolfi, F., and Giroud, M. 2003. Epidemiology of hemorrhagic strokes. J. Neuroradiol. 30: 291-297. [Medline]

8. Gong, C., Hoff, J.T., and Keep, R.F. 2000. Acute inflammatory reaction following experimental intracerebral hemorrhage in rat. Brain Res. 871: 57-65. [Medline] [CrossRef]

9. Kaur, J., Zhao, Z., Klein, G.M., Lo, E.H., and Buchan, A.M. 2004. The neurotoxicity of tissue plasminogen activator? $J$. Cereb. Blood Flow Metab. 24: 945-963. [Medline] [CrossRef]

10. MacLellan, C.L., Girgis, J., and Colbourne, F. 2004. Delayed onset of prolonged hypothermia improves outcome after intracerebral hemorrhage in rats. J. Cereb. Blood Flow Metab. 24: 432-440. [Medline] [CrossRef]

11. Matsushita, K., Meng, W., Wang, X., Asahi, M., Asahi, K., Moskowitz, M.A., and Lo, E.H. 2000. Evidence for Apoptosis After Intracerebral Hemorrhage in Rat Striatum. J. Cereb. Blood Flow Metab. 20: 396-404. [Medline] [CrossRef]

12. Mayer, S.A., Brun, N.C., Begtrup, K., Broderick, J., Davis, S., Diringer, M.N., Skolnick, B.E., and Steiner, T. 2008. Efficacy and safety of recombinant activated factor VII for acute intracerebral hemorrhage. N. Engl. J. Med. 358: 2127-2137. [Medline] [CrossRef]

13. Mendelow, A.D., Gregson, B.A., Fernandes, H.M., Murray, G.D., Teasdale, G.M., Hope, D.T., Karimi, A., Shaw, M.D., and Barer, D.H. 2005. Early surgery versus initial conser- 
vative treatment in patients with spontaneous supratentorial intracerebral haematomas in the International Surgical Trial in Intracerebral Haemorrhage (STICH): a randomised trial. Lancet 365: 387-397. [Medline]

14. Mendelow, A.D. and Unterberg, A. 2007. Surgical treatment of intracerebral haemorrhage. Curr. Opin. Crit. Care 13: 169-174. [Medline] [CrossRef]

15. Morgan, T., Zuccarello, M., Narayan, R., Keyl, P., Lane, K., and Hanley, D. 2008. Preliminary findings of the minimally-invasive surgery plus rtPA for intracerebral hemorrhage evacuation (MISTIE) clinical trial. Cerebral. Hemorrhage 105: 147-151. [CrossRef]

16. Narayanan, S. 1999. Multifunctional roles of thrombin. Ann. Clin. Lab. Sci. 29: 275-280. [Medline]

17. Park, H.K., Chu, K., Lee, S.T., Jung, K.H., Kim, E.H., Lee, K.B., Song, Y.M., Jeong, S.W., Kim, M., and Roh, J.K. 2005. Granulocyte colony-stimulating factor induces sensorimotor recovery in intracerebral hemorrhage. Brain Res. 1041: 125-131. [Medline] [CrossRef]

18. Rincon, F. and Mayer, S.A. 2004. Novel therapies for intracerebral hemorrhage. Curr. Opin. Crit. Care 10: 94-100. [Medline] [CrossRef]

19. Rosenberg, G.A., Mun-Bryce, S., Wesley, M., and Kornfeld, M. 1990. Collagenase-induced intracerebral hemorrhage in rats. Stroke 21: 801-807. [Medline] [CrossRef]

20. Sang, Y.H., Su, H.X., Wu, W.T., So, K.F., and Cheung, R.T. 2011. Elevated blood pressure aggravates intracerebral hemorrhage-induced brain injury. J. Neurotrauma 28: 25232534. [Medline] [CrossRef]

21. Song, E.C., Chu, K., Jeong, S.W., Jung, K.H., Kim, S.H., Kim, M., and Yoon, B.W. 2003. Hyperglycemia exacerbates brain edema and perihematomal cell death after intracerebral hemorrhage. Stroke 34: 2215-2220. [Medline] [CrossRef]
22. Steiner, T., Kaste, M., Forsting, M., Mendelow, D., Kwiecinski, H., Szikora, I., Juvela, S., Marchel, A., Chapot, R., Cognard, C., Unterberg, A., and Hacke, W. 2006. Recommendations for the management of intracranial haemorrhage - part I: spontaneous intracerebral haemorrhage. The European Stroke Initiative Writing Committee and the Writing Committee for the EUSI Executive Committee. Cerebrovasc. Dis. 22: 294-316. [Medline]

23. Sudlow, C.L. and Warlow, C.P. 1997. Comparable studies of the incidence of stroke and its pathological types: results from an international collaboration. International Stroke Incidence Collaboration. Stroke 28: 491-499. [Medline] [CrossRef]

24. Teernstra, O.P., Evers, S.M., Lodder, J., Leffers, P., Franke, C.L., and Blaauw, G. 2003. Stereotactic treatment of intracerebral hematoma by means of a plasminogen activator: a multicenter randomized controlled trial (SICHPA). Stroke 34: 968-974. [Medline] [CrossRef]

25. Tsirka, S.E., Rogove, A.D., and Strickland, S. 1996. Neuronal cell death and tPA. Nature 384: 123-124. [Medline] [CrossRef]

26. Tuhrim, S. 2008. Intracerebral hemorrhage-improving outcome by reducing volume? N. Engl. J. Med. 358: 21742176. [Medline] [CrossRef]

27. Wang, Y.F., Tsirka, S.E., Strickland, S., Stieg, P.E., Soriano, S.G., and Lipton, S.A. 1998. Tissue plasminogen activator (tPA) increases neuronal damage after focal cerebral ischemia in wild-type and tPA-deficient mice. Nat. Med. 4: 228231. [Medline] [CrossRef]

28. Woo, D. and Broderick, J.P. 2002. Spontaneous intracerebral hemorrhage: epidemiology and clinical presentation. Neurosurg. Clin. N. Am. 13: 265. [Medline] [CrossRef] 